\title{
The Difficulties, Opportunities and Challenges of Covid-19 by Chinese Medicine on China
}

\begin{abstract}
XUANXUAN WANG ${ }^{1}$, HUA LI $^{2}$, PING WANG ${ }^{1}$, XUN GONG ${ }^{3}$, GUANGJIE LI ${ }^{4}$, ZHENCHUAN JIANG ${ }^{5}$, JIAO CHEN $^{6}$, GUO LU $^{6}$, HONGPING CHENG ${ }^{7}$, ZHENG WANG ${ }^{8,9 *}$ AND XIAOWEI WU ${ }^{10}$

Department of Integrated TCM \& Western Medicine, Hubei Cancer Hospital, Tongji Medical College, Huazhong University of Science and Technology, Wuhan, ${ }^{1}$ Clinical College of Chinese Medicine, Hubei University of Chinese Medicine, Wuhan, ${ }^{2}$ Nursing Department of Cadre Sanatorium of Hainan \& Geriatric Hospital of Hainan (CSH), Haikou, ${ }^{3}$ Center for civil-military inosculation and development of new material industry in Huludao City, Huludao, ${ }^{4}$ Medical Service Division of Cadre sanatorium of Hainan \& Geriatric hospital of Hainan (CSH), Haikou, China, ${ }^{5}$ Graduate School of Lyceum of the Philippines University, Manila, Philippines, ${ }^{6}$ School of International Education, Hainan Medical University, Haikou, ${ }^{7}$ School of public health, Hubei Medical College, Shiyan, Hubei, ${ }^{8} \mathrm{Key}$ Laboratory of Environmental Pollution Monitoring and Disease Control, Ministry of Education, Guizhou Medical University, Guiyang, ${ }^{9}$ Research Center for Healthcare Management, School of Economic and Management, Tsinghua University, Beijing, ${ }^{10}$ Department of Thoracic Surgery, TongJi Hospital, TongJi Medical College, Huazhong University of Science and Technology, Wuhan, China
\end{abstract}

\section{Wang et al.: Opportunities and Challenges of Covid-19 by Chinese Medicine}

\begin{abstract}
Recently, Coronavirus disease $\mathbf{2 0 1 9}$ is the most frequent public health event in human history, spreads fastest, has the most extensive infection and the most difficult prevention and control, which has been abstracting the most impressive attention of the whole world. China, as the first outbreak place, has suffered seriously from the outbreak of Covid-19. The impact on domestic economy is profound and complex. How to effectively deal with once in a century global pandemic is still a world problem to be solved by the international community. Proceeding from the reality of China's national conditions, following the objective law of prevention and control of infectious diseases, China has made great efforts to explore a set of effective "Chinese methods" in the control of epidemic situation and treatment of patients. Investigation, detection and monitoring with epidemiological investigation as the core has become the key link to cut off the chain of virus transmission and implement timely treatment for patients. To set up hospitals at different levels and to treat patients with mild, moderate and severe diseases according to their conditions, the combination of traditional Chinese and Western medicine has greatly reduced the infection rate and death rate and improved the cure rate. From the whole industrial chain, the primary industry, the secondary industry and the tertiary industry are all almost affected. However, some difficulties, opportunities and challenges of Covid-19 should be paid attention to and grasped.
\end{abstract}

Key words: Coronavirus disease 2019, impact globally and domestically, difficulties, opportunity, Chinese medicine

In the beginning of the year of 2020, the planet is focusing on the globalized coronavirus, coronavirus disease 2019 (COVID-19). Until October 29 , the number of confirmed cases achieves more than 44002 003 cases and 1167988 in 152 countries around the world, according to the latest real time statistics from World Health Organization (WHO). As of October $28^{\text {th }}$, the United States (US) reported 8837688 cases of COVID-19 and 227421 cases died, those data released by Johns Hopkins University in the US. In May 2020, the novel coronavirus pneumonia epidemic was reported by the Asian Development Bank (ADB), which could cause losses of up to 5 trillion and 8.8 trillion dollars, equivalent to $6.4 \% \sim 9.7 \%$ of Gross domestic product

*Address for correspondence E-mail: yantuoleibu9965@163.com
(GDP $)^{[1]}$. In order to curb the spread of the virus, economies affected by the epidemic have closed borders, restricted travel and imposed blockades. In the short term and long term prevention and control situation, these measures may cause the global trade volume to decline by $\$ 1.7-2.6$ trillion $^{[2]}$. Global employment will decrease by 158 million to 242 million, of which the Asia Pacific region accounts for $70 \%$ of the total. The This is an open access article distributed under the terms of the Creative
Commons Attribution-NonCommercial-ShareAlike 3.0 License, which
allows others to remix, tweak, and build upon the work non-commercially,
as long as the author is credited and the new creations are licensed under
the identical terms

Accepted 18 February 2021

Revised 26 September 2020

Received 03 June 2020

Indian J Pharm Sci 2021;83(1):13-20 
world's labor income will decrease by $\$ 1.2$ trillion to 1.8 trillion, of which the loss of Asia Pacific economies will account for about $30 \%$, that is, $\$ 359$ billion to 550 billion. As the first outbreak country, China has already been fighting against the coronavirus more than 2 mo. The government locks down Wuhan, a 11 million population city and lockouts the most of industries and all school in the whole country. The whole industrial chain such as manufacturing, traveling, restaurants and other industries have suffered great damages ${ }^{[3]}$.

\section{CHINA ECONOMIC REALITY UNDER COVID-19}

Compared with the other two industries, the primary industry only accounting for $7.1 \%$ of the GPD in 2019, suffers small impact from the COVID-19. In recent years, the primary industry keeps slow but stable increasing, and is not an absolute factor in GDP growth $^{[4]}$.

The secondary industry is obviously affected, which means industrial production declines but the prices increases. Industrial added value falls $13.5 \%$ y on y. To be specific, the added value of state owned enterprises decreases by $7.9 \%$. Joint stock enterprises declines by $14.2 \%$, and foreign funded enterprises and Hong Kong, Macao Taiwan enterprises decrease by $21.4 \%$. Private businesses fall $20.2 \%$. The added value of the mining sector, the manufacturing sector and the electricity, heat, gas and water production and supply sectors falls by $6.5 \%, 15.7 \%, 7.1 \%$ respectively ${ }^{[5]}$.

However, some production of important materials and supplies still keep growing. The output of medical protection and living necessities increases rapidly, masks and alcohol increased by $127.5 \%$ and $15.6 \%$ respectively and the output of frozen meat and instant noodles climbing by $13.5 \%$ and $11.4 \%$ respectively. The growth of high tech products also keeps raising. The output of smart watches, smart bracelets, semiconductor discrete devices and integrated circuits increased by $119.7 \%, 45.1 \%, 31.4 \%$ and $8.5 \%$, respectively. In addition, the production of basic raw materials is stable, and the output of pig iron, crude steel, plate glass and ten non-ferrous metals increases by $3.1 \%, 3.1 \%$, $2.3 \%$ and $2.2 \%$, respectively. Until February $25^{\text {th }}$, $85.6 \%$ of large and medium sized manufacturing enterprises have returned to work and production and business activities are resuming in an orderly manner, according to the purchasing managers index (PMI) ${ }^{[5]}$.

The tertiary industry has been hit hard. In the first 2 mo of this year, the national index of service sector production falls $13.0 \%$ y on y. In terms of major industries, except the financial sector and the information sector grow by $4.5 \%$ and $3.8 \%$ respectively, other sectors experienced declines to varying degrees.

The total retail sales of consumer goods are 5213 billion yuan, declining by $20.5 \%$. By location, the statistics in cities and towns reaches 4.4881 trillion yuan, decreasing $20.7 \%$. The statistics in rural areas reaches 724.9 billion yuan, decreasing $19.0 \%$. By various types, the revenue of catering industry is 419.4 billion yuan, cutting down $43.1 \%$. Retail sales of commodities reached 4.7936 trillion yuan, cutting down $17.6 \%{ }^{[5]}$.

\section{MEASURES TAKEN BY THE CHINESE GOVERNMENT}

\section{Unify the public's awareness of diseases:}

COVID-19 should be recognized as a key factor in the early warning of the outbreak of the disease:

On the one hand, it is necessary to provide the public with continuous, clear and important information, so that residents can have a timely and comprehensive understanding of the government's policies and strategic measures in response to the epidemic situation and build a benign communication and interaction between the government and the public. On the other hand, based on the sense of social responsibility and trust in the government, the public actively participated in the nationwide fight against the epidemic ${ }^{[6]}$.

Timely, comprehensive, open and transparent release of epidemic information: Establish a daily news release mechanism to release the epidemic information as soon as possible. In more than $2 \mathrm{mo}$, the State Council Information Office and relevant departments held nearly 200 press conferences in the name of the joint prevention and control mechanism of the State Council and Hubei Province, where the epidemic is the most serious. In addition, Beijing and other provinces, autonomous regions and cities also held hundreds of local press conferences ${ }^{[7]}$. These live news conferences fully informed the latest progress of the epidemic situation and anti-epidemic work and fully responded to the concerns of public opinion at home and abroad.

We will start the daily report system of epidemic data and provide accurate classification information in a timely and comprehensive manner: On the official website and other government affairs platforms, the National Health Commission reports daily that new 
confirmed cases, new cured and discharged cases, new severe cases, new death cases, new suspected cases, cumulative reported confirmed cases, cumulative cured and discharged cases, cumulative death cases, existing suspected cases, cumulative traced close contacts and asymptomatic infected persons are related data.

Update the "epidemic map" in real time. The "epidemic map" released by big data technology is used to indicate the specific location, distance and number of people of the epidemic spreading through the name, address and location of the community. The emergency response and prevention and control alarm mechanism are constantly adjusted according to the situation, and the traffic control and other measures are published in a timely manner, so as to facilitate the public to more effectively prevent infection and curb the spread of the virus $^{[8-10]}$.

\section{Popularize scientific knowledge of prevention and} control: To organize scientists and scientific research institutions to continuously release authoritative opinions and professional opinions and suggestions on scientific prevention and control through various press conferences, media interviews and the Internet on the basis of solid research, so as to improve the public's scientific cognition level, guide scientific prevention and control, and advocate scientific killing, especially to promote the popularization of the simplest and most effective public protection such as wearing masks, washing hands frequently and ventilation frequently, measures to enhance public self-protection ability. The prevention and control guidelines with strong operability shall be issued to provide specific guidance to the public.

COVID-19 handbook and COVID-19 public protection guide is issued by the National Health Committee, from personal and family protection, home medical observation, rational medical treatment, psychological counseling and other aspects, so that the public can effectively master the protection skills and means ${ }^{[11]}$.

COVID-19 infection emergency guidelines for psychological crisis intervention were promulgated by the National Health Committee and classified intervention was implemented. The joint prevention and control mechanism of the State Council issued the "notice on the establishment of psychological assistance hotline to cope with epidemic situation", and all localities set up psychological assistance hotline in response to epidemic situation on the basis of the original psychological assistance hotline. Colleges and universities have opened a psychological support hotline and network counseling service. Internet hospitals and related enterprises and institutions provide online and telephone hotline and other social psychological services $^{[12]}$.

Mainstream media provide public opinion support: The news media have fully reported the effectiveness of joint prevention and control measures in various regions and departments, so that the people can always understand what the party and the government are doing and what to do. It should fully reflect the stories of fighting against the epidemic, especially the front line medical personnel, community workers, volunteers and police and resolve the anxiety and fear of the public. For example, high density release of authoritative information comprehensively reflects major issues of public concern, such as anti-epidemic relief, material support, vaccine development and social donations. Special areas were set up on various platforms to interpret the epidemic situation, analyze the data, study and judge the trend and timely refute rumors.

\section{Participation of the whole people and formation of} social joint forces: Combined with the experience of fighting severe acute respiratory syndrome (SARS) in 2003, no matter the long term "city closure" in key areas, the implementation of community access management, the whole people's home isolation, the "14 d" self-isolation under specific circumstances, as well as the family and personal hygiene habits and social communication restrictions, laid the foundation for effectively reducing the virus infection rate. Community "grid management" is the key factor to curb the spread of the epidemic. As a kind of social management mechanism, community network, with the cooperation of volunteers, has made indepth visits to 650000 urban and rural communities, preached epidemic prevention knowledge, conducted psychological counseling, received express delivery, and purchased daily necessities on behalf of 4 million urban and rural community workers, so that the support and security services for the people directly entered hundreds of millions of families.

Resource allocation and material support: In order to lay an important material foundation for the fight against the epidemic situation, we should allocate resources efficiently, optimize the organization of production, strengthen the emergency supply of medical materials and necessities, strictly investigate and deal with illegal 
acts of manufacturing and selling counterfeit goods and win the logistics support war.

Concentrate the strength of medical expert team: We will concentrate national expert resources to guide the whole chain of prevention and treatment. China's top respiratory and critical care medicine experts gathered in Wuhan to intervene in the early stage of the epidemic. At the same time, we should actively explore new drugs and new therapies and timely incorporate effective treatment methods into the diagnosis and treatment plan and promote them to the national treatment work.

Enhance the production and supply of medical materials and medical support services: Relying on a complete industrial production system, medical enterprises overcome the difficulties of insufficient workers returning to work, resume the production of medical supplies as soon as possible, and maximize the production capacity. With the rapid adjustment of the production conditions of the epidemic prevention and control equipment, the enterprises have adjusted the production conditions of the epidemic prevention and control equipment and equipment. In a short period of time, including disposable medical masks, medical surgical masks and medical KN95 masks, China's daily output of masks exceeded 110 million; the daily production capacity of disposable medical protective clothing exceeded 1.5 million pieces; the daily output of hand held infrared thermometers reached 400000 ; and the daily supply of kits reached nearly 350000 in early $\operatorname{March}^{[13]}$.

At the same time, the supply chain and logistics channels should be unblocked and the cooperation mechanism of joint guarantee and supply should be established to continuously transport the support materials from all over the country to Hubei Province. Within 1 mo, the daily dispatching supply of medical protective clothing increased from 21000 pieces to 270000 pieces and the daily dispatching supply of KN95 masks increased from 72000 pieces to 562000 pieces. We will provide services such as remote consultation, expert live broadcast and medical training. Making full use of remote visual medical system and artificial intelligence (AI) assisted surgical equipment based on 5G network cannot only partly solve the medical needs of the public for other diseases during the epidemic period, but also prevent hospital cross infection to the maximum extent.

The National Health Commission issued a series of technical documents and relevant requirements, standardized the use of protective equipment and protection process, implemented the principles of hospital regional setting and "three areas and two channels" for respiratory diseases, greatly reduced the infection rate of medical staff, and realized "zero infection" for more than 40000 medical personnel in Hubei Province.

Coordinate the security of living materials to ensure the safety of daily necessities and timely delivery to resident's homes. Grid management is adopted to deliver goods to the house directly to ensure daily life supplies. Vegetables that have been quarantined and meet the epidemic prevention standards are sent directly to the community through non-contact distribution, which relieves the resident's worries about living security and epidemic prevention safety. Make full use of Internet technology and popularize "contactless consumption" in an all-round way. The advanced Internet plus logistics distribution and e-commerce platform guarantees all links of the national material ordering and distribution during the epidemic period. People can complete online purchase and offline receiving through mobile phone, and logistics, express is delivered through property trusteeship, fixed point handover and self-holding cabinet, so as to avoid the risk of infection, goods and funds coming to the supermarket, shopping malls and farmers market. Under the Internet infrastructure, the whole industrial chain, such as transportation and delivery, operates like a gear, realizing cash free payment for commodity sales and zero contact delivery for logistics distribution.

Crowd isolation and traffic control: In the absence of vaccines and specific drugs, non-medical intervention based on traditional isolation is the most basic and effective means to stop the spread of major epidemic. Unconventional social isolation measures are flexible and humanized social control have blocked the large scale spread of the virus and become the most critical factor to curb the spread of the epidemic in the country.

The traffic in the disaster areas was closed and controlled to cut off the route of virus transmission to the maximum extent: The operation of ferries, long-distance passenger transport, airports and railway stations in Wuhan and Hubei will be suspended and the departure of waterway passenger transport lines on the roads into Wuhan will be suspended nationwide. 1501 traffic control checkpoints were set up at highway toll stations and county and village road junctions in provinces, cities and cities including 51 in Wuhan and 
1450 in other cities and prefectures, so as to prevent the virus from spreading to the rural areas with weak health infrastructure in China and the province. From January 23, Wuhan, a super large city with 11 million permanent residents, was closed for $76 \mathrm{~d}$. Greatly reduce the flow of people in the city and the possible spread of the virus ${ }^{[14]}$.

Implementation of classified traffic control in non-epidemic areas: In addition to Hubei Province, according to the list of low-risk, medium-risk and highrisk counties (cities, districts and banners) under their jurisdiction, other regions in China have implemented accurate prevention and control at different levels, and dynamically control urban and rural road transport services such as road passenger transport urban public transport (including urban rail transit) and taxi (including online taxi).

Avoid crowd gathering and cross infection by various means: Close cinemas, theaters, Internet cafes and gymnasiums, open public service places such as stations, airports, docks, farmers markets, shopping malls, supermarkets, restaurants, hotels and other public service places, as well as closed means of transportation such as cars, trains and airplanes. Measures such as environmental sanitation treatment, disinfection, ventilation and flow restriction shall be implemented. The entry personnel must take temperature, wear masks and avoid no cross infection. The implementation of home office, the implementation of distance education, cancel or postpone various public gathering activities, flexible working methods such as online office, off peak work and on duty in turn are adopted.

According to the actual situation of the epidemic, various localities postponed the opening time of school, suspended all offline courses of training institutions, replaced classroom teaching with online teaching, and effectively controlled the flow and aggregation of 300 million school personnel in China.

\section{Inspection and dynamic monitoring:}

Comprehensive investigation by community: For example, Wuhan launched two rounds of centralized screening. In view of the problem, that more than 80 $\%$ of the epidemic situation is spread in the community, a carpet investigation is carried out by means of door to door investigation and self-examination and self-report based on the community grid. More than 80000 volunteers registered, more than 4 million residents in the city, including confirmed patients, suspected patients, fever patients who could not be excluded from infection, close contacts of confirmed patients, and people returning from key epidemic areas through household, telephone and Internet means.

Improve detection level: Enhance the supply capacity of kits. At the same time, we will expand the testing institutions, allow the tertiary hospitals, centers for Disease Control and professional testing institutions with the conditions of "secondary biosafety laboratory" and "reverse transcription polymerase chain reaction (RT-PCR)" conditions to carry out nucleic acid detection, and provide $7 \times 24 \mathrm{~h}$ uninterrupted detection service to realize the comprehensive detection of suspected cases.

Establish dynamic management system: The strategy of prevention and control has been implemented in the whole country, with counties as the unit, and according to the comprehensive analysis and judgment of population and disease incidence, the low, medium and high epidemic risk levels are divided. With the change of the epidemic situation and the increase of asymptomatic infections, it was decided to take $14 \mathrm{~d}$ of centralized isolation medical observation for the asymptomatic infected persons and the isolation could be lifted only if the nucleic acid test of the samples was negative for two consecutive times.

Launch "Health Code" mode to ensure "green travel": Residents download the "Health Code" on their mobile phones through the government service network platform, which is used as the certificate for travel, return to work, production and school, daily life and access to public places. According to the three colors of "Health Code" green, yellow and red, the traffic control and classified disposal are carried out to realize the accurate collection and statistical analysis of epidemic control data. According to the flow of personnel, the accurate identification, precise implementation and precise prevention and control of zoning and classification are realized, so that the safety risk is "transparent" and the public travel is more assured $^{[15,16]}$.

Carry out in-depth flow adjustment work: Establish an epidemiological investigation team to accurately track and cut off the route of virus transmission. Relying on big data and information system, improve the accuracy and efficiency of flow regulation, take the 
tracking of close contacts as an important link, realize early, fast and complete case detection, and carry out medical observation on asymptomatic infected persons and close contacts, and reduce the risk of infection. During the peak period of the epidemic, 1800 flow control teams with 5 members were set up in Wuhan to comprehensively investigate the track of confirmed and close contacts, and carry out tracing and investigation of the source of infection.

Diagnosis and treatment plan and treatment capacity: At the same time, free treatment is implemented to relieve the worries of the patients, so as to avoid serious illness and death and expand the source of infection, greatly reduce the infection rate and death rate and improve the cure rate.

Establish the dual goal of unifying the control of infectious source and improvement of treatment: "Centralized treatment" and "bed expansion" combined and all patients were admitted. In Wuhan, the hardest hit area of the epidemic, on the one hand, referring to the model of building "Xiaotangshan Hospital" in Beijing to fight against SARS and completed the construction of "Huoshen mountain" and "Leishen mountain" infectious diseases specialized hospitals with a capacity of more than 1000 beds in about $10 \mathrm{~d}$ and put them into use. A number of "shelter hospitals" were built, a number of designated hospitals were rebuilt and expanded, and a number of comprehensive medicines were transformed. In a short period of time, more than 100000 beds have been added in the hospital, which has realized the significant transformation from "people waiting for beds" to "beds and others", and has solved the problem of large-scale admission and treatment in a short period of time ${ }^{[17]}$.

We should distinguish the light and severe patients, and construct the classified and classified treatment system composed of "shelter hospital" and "designated hospital". Forty six hospitals in Wuhan were equipped with artificial membrane lung ventilator and other equipment, and the oxygen supply pipeline was reformed to improve the treatment ability and reduce the death rate. At the same time, relying on the "shelter hospital" to treat patients with mild illness, large-scale isolation treatment was done. Novel coronavirus pneumonia patients in 15 hospitals account for more than $1 / 4$ of the new crown pneumonia patients, accounting for more than 1.2 million.

Improve the treatment plan and optimize the treatment means: Individualized treatment was adopted according to the severity of symptoms. Patients with mild illness were admitted to the shelter hospital in a unified way, and comprehensive treatment measures such as antiviral, oxygen inhalation, Traditional Chinese medicine (TCM) and so on were clarified to prevent mild illness from turning into severe disease. For more than $80 \%$ of severe patients complicated with serious basic diseases, "one person, one policy" was implemented. We should establish a multi-disciplinary consultation system for infection, respiration, severe disease, heart and kidney, formulate nursing standards for severe and critical patients, introduce measures such as high flow oxygen inhalation, noninvasive and invasive mechanical ventilation, prone position ventilation, and attach importance to the discussion system of death cases, so as to ensure scientific treatment, effectively reduce the mortality rate and improve the cure rate.

Adhere to the combination of clinical and scientific research, strengthen drug screening and vaccine research and development. Actively promote scientific and technological research, screen effective prescriptions, timely feedback curative effect, test, research, and promote fabiravir, chloroquine phosphate, plasma in convalescent period, etc., especially in view of the fact that there is no specific drug available and guided by the treatment demand, screen out the clinical effective drugs such as Jinhua Qinggan granules, Lianhua Qingwen capsules and granules, Qingfei Paidu decoction, etc. In group A, clinical trials were started with new crown vaccine and new crown inactivated vaccine. Breakthroughs were made in plasma therapy, stem cell therapy and other technologies. Diagnosis and treatment programs were continuously improved and the proportion of patients from mild to severe diseases was significantly reduced. The cure rate of patients increased from $14 \%$ in the initial stage to $93 \%$.

\section{Traditional Chinese medicine treatment has obvious} advantages: TCM team participated in the treatment. TCM early intervention, whole process participation and carried out classified treatment for the patients with mild illness, early intervention and early use of TCM; for patients with severe and critical illness, the combination of TCM and Western medicine is given. Chinese medicine doctors participate in the whole process of treatment plan formulation; medical observation of fever patients and close contacts taking TCM to improve immunity; implementation of Chinese medicine rehabilitation program for discharged patients. 
COVID-19 treatment system with high utilization rate and high efficiency has become a major feature of China's clinical treatment for COVID-19. The utilization rate and total effective rate of TCM were over $90 \%$. Clinical efficacy observation shows that TCM can effectively relieve symptoms, reduce the development of light and common type to severe type, improve the cure rate reduce the death rate and promote the recovery of the body of people in recovery period.

China Academy of Chinese medicine novel coronavirus pneumonia hospital of Guanganmen hospital, Liu Baoyan team of Chinese Academy of Chinese Medical Sciences, Beijing University of Chinese Medicine and Liu Jianping team analyzed 1476 cases of new crown pneumonia in Wuhan integrated traditional Chinese and Western medicine hospital. After taking into account factors such as gender and age, hierarchical analysis and propensity score matching were used respectively. The results showed that after adjusting for eight factors, such as age, gender, combined basic diseases and treatment course, comprehensive intervention of TCM was the independent influencing factor of nucleic acid recovery.

\section{FOCUSING ON THE OPPORTUNITY}

Now, the whole world is facing a great challenge but we need establish the will to beat this no fire war and realize the opportunity. Some positive signal cannot be ignored. In February, the index of business activity in the service sector is $30.1 \%$, among which the index of business activity in the financial sector is $50.1 \%$, remaining in the expansion range. The index of business activity in the telecom and Internet software sectors are $43.3 \%$ and $41.4 \%$, respectively, higher than the index of business activity in the service sector by 13.2 and 11.3 percentage points and significantly better than the overall level of the service sector ${ }^{[18]}$.

Therefore, it can be concluded that the outbreak may be positive for some of China's advantage industries. Industries such as communications, big data, cloud computing, the Internet and medicine have played an obvious role during the epidemic. The changes in user behavior is expected to continue after the outbreak ends, which may promote the further development of the relevant advantageous industries.

The positive role of the Internet industry has been fully reflected. For example, the online video industry has partly replaced the consumption of films in cinemas, while e-commerce has offset the shortage of offline consumption. Online education has replaced offline classes and teleconferencing and telecommuting systems have helped companies get back to work.

In addition, right now, the domestic situation of the Covid-19 has been basically controlled, the resumption of work and production has been completed. While in Europe, the U.S and other developed countries are showing an outbreak trend. From the perspective of global production, it may cause the capital of relevant countries to seek a safer haven destination. China is one of the major choices for international capital and related industries in terms of market capacity, industrial maturity, human resources, epidemic prevention and control situation. Therefore, the current situation of epidemic development may lead to further international industry to our country.

Furthermore, a great amount of medical supplies is generated by the global outbreak. Based on China's advanced manufacturing, the increasing number of medical supplies are exporting to the rest of world such as breathing machine, facial mask, protection suits. At the same time, it is a good chance to improve the globalization of Renminbi (RMB).

It is also a good opportunity for China to update industrial structure, AI will play more important role in various industries. Obviously, AI combined with medical treatment will be a trend in recent years. AI medical imaging technology has been able to complete the preliminary computed tomography (CT) screening work well, greatly reducing the working pressure of first line doctors. So that medical resources have been more effective and accuratly used.

The big data has played an important role in the prevention and control of the COVID-19. A shared platform has been established by governments at all levels to collect, gather and analyze data through the big data. In the shortest time, it has collected transportation, disease control, medical supplies, assistance, supervision, logistics and other departments to fight against the epidemic. At the same time, by big data analysis, the change trend and internal evolution of the epidemic can be grasped. The government can strengthen the epidemiological investigation work form, risk prevention and control, effectively prevent the spread of the coronavirus.

The impact of the outbreak of COVID-19 on China's economy is profound and complex. The new epidemic has brought about a serious negative impact on China's economy. However, at the same time, the epidemic has also stimulated the creativity of the whole people 
to respond to emergencies and the transformation and upgrading of consumer services, e-commerce and other related industries have been accelerated. From the global perspective, the government's effective outbreak control provides an opportunity for China to demonstrate its governance to the international community, which helps to improve the country's image. It has demonstrated the superiority of China's political and economic system through promptly control the spread of the epidemic in China and restoring the order of production and operation.

Proceeding from the reality of China's national conditions, following the objective, law of prevention and control of infectious diseases, China has made great efforts to explore a set of effective Chinese methods in the control of epidemic situation and treatment of patients. Investigation, detection and monitoring with epidemiological investigation as the core has become the key link to cut off the chain of virus transmission and implement timely treatment for patients, to set up hospitals at different levels and to treat patients with mild, moderate and severe diseases according to their conditions. The combination of TCM and Western medicine has greatly reduced the infection rate and death rate, and improved the cure rate. From the whole industrial chain, the primary industry, the secondary industry, and the tertiary industry is almost affected. However, some difficulties, opportunities and challenges of COVID-19 should be paid attention to and grasped.

\section{Acknowledgements:}

We would like to thanks Dr. Zhenchuan Jiang, Dr. Jiao Chen, for having prepared the vehicle used in the study. We are grateful to Dr. Zheng Wang for reviewing the manuscript.

\section{Funding:}

This research was supported by youth program of high-end science and technology innovation think tank of Chinese Association for Science and Technology (DXB-ZKQN-2017-043).

\section{Conflict of Interests:}

The authors declared no conflict of interest.

\section{REFERENCES}

1. Novel Coronavirus (COVID-19) Situation. World Health Organization; 2020.

2. The annual growth rate of global GDP in 2020 will be reduced by about $0.2 \%$ due to the COVID-19. Inewsweek; 2020.

3. COVID-19 Outbreak to have significant economic impact on developing Asia. Asian Development bank; 2020.

4. GDP data in China. Eastmoney; 2020.

5. From January to February, the national economy withstood the impact of the new crown pneumonia epidemic. National Bureau of Statistics; 2020.

6. https://www.360kuai.com/pc/detail?url=http $\% 3 \mathrm{~A} \% 2 \mathrm{~F}$ $\% 2$ Fzm.news.so.com\%2F 703 f6707c25a37e9a619dad a 328905 eb\&check $=39$ a 662 dd000b42 $1 \mathrm{f} \&$ sign $=360 \_5$ $57 \mathrm{c} 3 \mathrm{bbd} 1 \&$ uid $=\mathrm{g} 1584242435980492084 \& \mathrm{dj}$ source $=\& \overline{\mathrm{tj}}_{-}$ url $=9 \mathrm{f} 217 \mathrm{~d} 2 \mathrm{bc} 4 \mathrm{ec} 3 \mathrm{dbb} 8$

7. Xu XF. The Three "circuit breakers" of the America Stock Market. 2020.

8. https://money.cnn.com/data/markets/

9. Tu GS. Analysis of the impact of the global spread of the Covid-19 on the Chinese economy. 2020.

10. ht t p://www. xinhuanet.com/fortune/202003/13/c_1125707126.htm

11. Brief Introduction of International Trade in 2019 December. Mofcom; 2020.

12. Jiang Z, Gong X, Cheng H. Research on Trading Friction between China and the US from Game Theory Perspective. Am J Ind Bus Manag 2020;10(01):19.

13. Trade between China, Japan and the South Korea exceeded 720 billion us dollars in 2018. Sina.com; 2019

14. In 2018, the total import and export volume of China and Europe reached US $\$ 682.2$ billion, an increase of $10.6 \%$. ocn. com; 2019.

15. China: Exports, percent of GDP. The GlobalEconomy; 2020.

16. Covid-19's impact on various industries. Sina Finance; 2020.

17. The impact of the Covid-19 on Traveling. Suning Finance; 2020.

18. How the Covid-19 influence China's economy. News Sina; 2020 . 